\title{
Anti-inflammatory Constituents from Solanum nigrum
}

\author{
Xing-Fu Cai, Young-Won Chin, Sei-Ryang Oh, Ok-Kyung Kwon, Kyung-Seop Ahn, and Hyeong-Kyu Lee* \\ Immune Modulator Research Center, Korea Research Institute of Bioscience and Biotechnology, Daejeon 305-806, Korea \\ *E-mail:hykylee@kribb.re.kr \\ Received October 14, 2009, Accepted November 6, 2009
}

Key Words: Solanum nigrum, Spirostane, Spirost-5-ene-3 $\beta, 12 \beta$-diol, Phenylpropanoids, Leukotriene $\mathrm{C}_{4}$

The whole plant of Solanum nigrum L. (Solanaceae) has been used as a folk medicine in Asian countries for the treatment of inflammation, edema, and mastitis. ${ }^{1,2}$ Chemical constituents of this plant have been reported to comprise alkaloids, glycoproteins, flavonoids, polyphenols, and triterpenoids. ${ }^{3,4}$ According to the literatures, a wide range of bioactivities including anti-inflammatory, ${ }^{5}$ antioxidant, ${ }^{6}$ antinociceptive, ${ }^{5}$ antipyretic, ${ }^{5}$ antitumor, ${ }^{7,8}$ antiulcerogenic, ${ }^{9}$ cancer chemopreventive, ${ }^{10}$ hepatoprotective, ${ }^{11}$ and immunomodulatory ${ }^{8}$ effects were observed while the active constituents responsible for these activities were not fully resolved so far. In the present study, the structure elucidation of a new compound along with 14 known compounds and their inhibitory activities on leukotriene $\mathrm{C}_{4}$ release are described.

The $50 \%$ EtOH extract of the fruits of S. nigrum was partitioned successively into ethyl acetate and water. The ethyl acetate-soluble fraction was purified by silica gel, reversedphase $\mathrm{C} 18$ gel column chromatography yielded one new compound 1 together with 14 known compounds, $N$-trans-feruloyltyramine (2), ${ }^{12}(R)$-3-(4-hydroxy-3-methoxyphenyl)- $N$-[2-(4hydroxyphenyl)-2-methoxyethyl]acrylamide $(3),{ }^{13}(E)$-ethyl caffeate (4), ${ }^{14}$ ethyl 4-hydroxy-3-methoxycinnamate (5), ${ }^{15}$ guaiacylglycerol- $\beta$-ferulic acid ether $(6),{ }^{16} 3$ - $O$-acetylbetulinic acid (7), ${ }^{17}$ chlorogenic acid (8), ${ }^{18}$ caffeic acid (9), ${ }^{19}$ methylsinapate (10), ${ }^{20} \beta$-sitosterol (11), ${ }^{21}$ drummondol (12), ${ }^{22,23} 2 \alpha, 9$-dihydroxy-1,8-cineole (13), ${ }^{24}$ tryptophol acetate (14), ${ }^{25}$ and 4-amino3-methoxyphenol (15) ${ }^{26}$ (Figure 1).

Compound 1 was obtained as an amorphous powder and its molecular formula was assigned as $\mathrm{C}_{26} \mathrm{H}_{40} \mathrm{O}_{4}$, based on the $[\mathrm{M}-\mathrm{H}]^{-}$peak at $\mathrm{m} / \mathrm{z} 415.2859$ (calcd for $\mathrm{C}_{26} \mathrm{H}_{39} \mathrm{O}_{4} 415.2848$ ) in the HRESIMS. The ${ }^{1} \mathrm{H}$ NMR spectrum showed two tertiary methyl groups at $\delta_{\mathrm{H}} 0.78(3 \mathrm{H}, \mathrm{s}, \mathrm{H}-18)$ and $1.06(3 \mathrm{H}, \mathrm{s}, \mathrm{H}-19)$, one secondary methyl at $\delta_{\mathrm{H}} 1.03(3 \mathrm{H}, \mathrm{d}, J=6.4 \mathrm{~Hz}, \mathrm{H}-21)$, an olefinic proton at $\delta_{\mathrm{H}} 5.35(1 \mathrm{H}, \mathrm{d}, J=5.1 \mathrm{~Hz}, \mathrm{H}-6)$, assignable to a spirostane moiety. ${ }^{27}$ The ${ }^{13} \mathrm{C}$ and DEPT NMR spectroscopic data showed three methyls at $\delta_{\mathrm{C}} 11.6,14.0$, and 19.9, two olefinic carbons at $\delta_{\mathrm{C}} 122.4$ and 142.3 , three oxygenated methines at $\delta_{\mathrm{C}} 72.4,80.4$, and 82.3 , one oxygenated methylene at $\delta_{\mathrm{C}}$ 63.3 , and one spiroketal carbon at $\delta_{\mathrm{C}} 110.6$. From ${ }^{1} \mathrm{H},{ }^{13} \mathrm{C}$, and DEPT NMR data, it was observed that a methyl group corresponding to $\mathrm{C}-27$ in $\mathrm{F}$ ring was absent in this molecule when compared with the typical spirostane-type compound. ${ }^{27}$ In the HMBC experiment, the presence of a double bond $\left(\Delta^{5,6}\right)$ in the $\mathrm{B}$ ring was determined by the observation of long range correlations between the methyl proton at $\delta_{\mathrm{H}} 1.06(\mathrm{Me}-19)$ and the olefinic carbon at $\delta_{\mathrm{C}} 142.3(\mathrm{C}-5)$, as well as the olefinic proton at $\delta_{\mathrm{H}} 5.35(\mathrm{H}-6)$ and $\delta_{\mathrm{C}} 38.0(\mathrm{C}-10)$ as shown in Figure 2. $\mathrm{HMBC}$ correlations of $\mathrm{H}-18\left(\delta_{\mathrm{H}} 0.78\right)$ to both of C-12 and C-13 were able to locate a hydroxy group on $\mathrm{C}-12$. The relative configuration of the hydroxy group at C-12 was assigned as $\beta$ orientation, based on the observed ROESY correlations of $\mathrm{H}-12$ to $\mathrm{H}-14$ and $\mathrm{H}-17$, and $\mathrm{H}-17$ to $\mathrm{H}-12, \mathrm{H}-14$ and $\mathrm{H}_{3}-21$. The ROESY correlations of $\mathrm{H}_{2}-26$ to $\mathrm{H}-16$, and $\mathrm{H}-16$ to $\mathrm{H}-17$, and $\mathrm{H}-17$ to $\mathrm{H}-12$ confirmed the relative configuration of the $\mathrm{C}-22$ position as $R^{*}$. By comparing the coupling constant of $\mathrm{H}-3$ $\left(\mathrm{W}_{1 / 2}=24 \mathrm{~Hz}\right)$ with the published value $\left(\mathrm{W}_{1 / 2}=24 \mathrm{~Hz}\right),{ }^{28}$ the hydroxy group on $\mathrm{C}-3$ was found to be $\beta$-oriented. Thus, the structure of 1 was determined to be a spirost-5-ene-3 $\beta, 12 \beta$-diol.
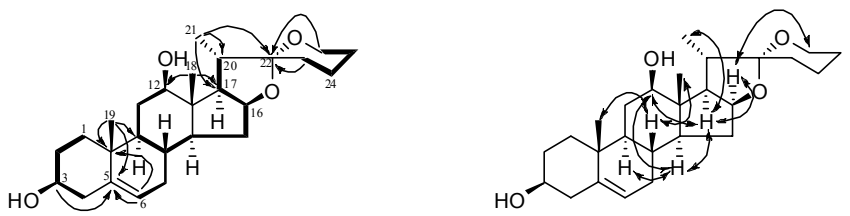

Figure 2. Key ${ }^{1} \mathrm{H}^{1}{ }^{1} \mathrm{H} \operatorname{COSY}(\longrightarrow), \operatorname{HMBC}(\mathrm{H} \longrightarrow \mathrm{C})$ and ROESY $(\longleftrightarrow)$ correlations of compound $\mathbf{1}$.

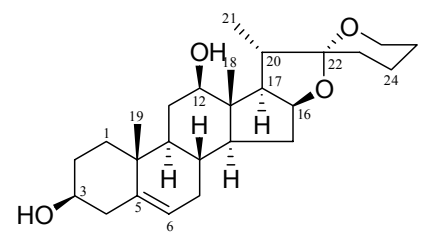

1<smiles>[R]C(CNC(=O)/C=C/c1ccc(O)c(OC)c1)c1ccc(O)cc1</smiles>

$\mathrm{R}$

$\mathrm{H}$ $\mathrm{OCH}_{3}$<smiles>[R2]c1ccc(/C=C/C(=O)OCC)cc1[R2]</smiles>

$\begin{array}{lll} & \mathrm{R}_{1} & \mathrm{R}_{2} \\ 4 & \mathrm{OH} & \mathrm{OH}\end{array}$

Figure 1. Structures of 1-5. 
Table 1. ${ }^{1} \mathrm{H}-(400 \mathrm{MHz})$, and ${ }^{13} \mathrm{C}-\mathrm{NMR}(100 \mathrm{MHz})$ Chemical Shifts of Compound 1 in $\mathrm{MeOH}-d_{4}$

\begin{tabular}{|c|c|c|}
\hline \multirow{2}{*}{ position } & \multicolumn{2}{|c|}{1} \\
\hline & $\delta_{\mathrm{C}}$ & $\delta_{\mathrm{H}}(J$ in $\mathrm{Hz})$ \\
\hline 1 & $38.6 \mathrm{t}$ & $1.08,1.86(\mathrm{~m})$ \\
\hline 2 & $32.3 \mathrm{t}$ & $1.53,1.81(\mathrm{~m})$ \\
\hline 3 & $72.4 \mathrm{~d}$ & $3.39\left(\mathrm{~m} \mathrm{~W}_{1 / 2} 24\right)$ \\
\hline 4 & $43.0 \mathrm{t}$ & $2.24(\mathrm{~m})$ \\
\hline 5 & $142.3 \mathrm{~s}$ & - \\
\hline 6 & $122.4 \mathrm{~d}$ & $5.35(\mathrm{~d}, 5.1)$ \\
\hline 7 & $31.8 \mathrm{t}$ & $1.62(\mathrm{~m})$ \\
\hline 8 & $31.9 \mathrm{~d}$ & $1.46(\mathrm{~m})$ \\
\hline 9 & $51.4 \mathrm{~d}$ & $1.08(\mathrm{~m})$ \\
\hline 10 & $38.0 \mathrm{~s}$ & - \\
\hline 11 & $32.4 \mathrm{t}$ & $1.39(\mathrm{~m})$ \\
\hline 12 & $80.4 \mathrm{~d}$ & $3.28(\mathrm{dd}, 6.7,4.5)$ \\
\hline 13 & $47.0 \mathrm{~s}$ & - \\
\hline 14 & $56.5 \mathrm{~d}$ & $1.10(\mathrm{~m})$ \\
\hline 15 & $33.0 \mathrm{t}$ & $2.01(\mathrm{~m})$ \\
\hline 16 & $82.3 \mathrm{~d}$ & $4.41(\mathrm{q}, 7.3,7.3,7.3)$ \\
\hline 17 & $63.3 \mathrm{~d}$ & $1.90(\mathrm{~m})$ \\
\hline 18 & $11.6 \mathrm{q}$ & $0.78(\mathrm{~s})$ \\
\hline 19 & $19.9 \mathrm{q}$ & $1.06(\mathrm{~s})$ \\
\hline 20 & $43.8 \mathrm{~d}$ & $1.89(\mathrm{~m})$ \\
\hline 21 & $14.0 \mathrm{q}$ & $1.03(\mathrm{~d}, 6.4)$ \\
\hline 22 & $110.6 \mathrm{~s}$ & - \\
\hline 23 & $31.7 \mathrm{t}$ & $1.71(\mathrm{~m})$ \\
\hline 24 & $31.7 \mathrm{t}$ & $1.71(\mathrm{~m})$ \\
\hline 25 & $24.9 \mathrm{t}$ & $1.92(\mathrm{~m})$ \\
\hline 26 & $63.0 \mathrm{t}$ & $3.76(\mathrm{~m})$ \\
\hline
\end{tabular}

Assignments were based on ${ }^{1} \mathrm{H},{ }^{13} \mathrm{C}$, DEPT, ${ }^{1} \mathrm{H}-{ }^{1} \mathrm{H}$ COSY, HMQC and HMBC experiments.

Table 2. Inhibition of LTC 4 -Release by Compounds 1-14 Obtained from the Fruits of $S$. nigrum $^{a, b}$

\begin{tabular}{cc}
\hline & $\mathrm{IC}_{50}(\mu \mathrm{M})$ \\
\cline { 2 - 2 } compound & $\mathrm{RBL}-1$ \\
\hline $\mathbf{1}$ & $22.4 \pm 0.1$ \\
$\mathbf{3}$ & $16.6 \pm 1.2$ \\
$\mathbf{4}$ & $22.6 \pm 1.3$ \\
$\mathbf{5}$ & $2.2 \pm 1.2$ \\
zileuton $^{c}$ & $10.3 \pm 1.3$ \\
\hline
\end{tabular}

${ }^{a}$ The results are means \pm standard deviation of three independent replicates. ${ }^{b}$ Compounds $(6-14)$ were found to be inactive $\left(\mathrm{IC}_{50} 25>\mu \mathrm{M}\right) .{ }^{c} \mathrm{~A}$ positive control.

Spirostane-type compounds with $\mathrm{C}_{26}$ have not been reported from natural sources so far while a few steroids with $\mathrm{C}_{26}$ have been found in the marine organisms. ${ }^{29,30}$

All the compounds obtained from the fruits of $S$. nigrum were evaluated for their inhibitory activities on $\mathrm{LTC}_{4}$ release, and the results were summarized in Table 2. Of the compounds tested, compound $\mathbf{4}$ exhibited the most potent inhibition of $\mathrm{LTC}_{4}$ release with an $\mathrm{IC}_{50}$ value of $2.2 \mu \mathrm{M}$ (the positive control, zileuton, $0.75 \mu \mathrm{M}$ ). Compound 5 , an analog of $\mathbf{4}$, also was found to inhibit $\mathrm{LTC}_{4}$-release $\left(\mathrm{IC}_{50} 10.3 \mu \mathrm{M}\right.$ ), consistent with the literature. ${ }^{31}$ The new compound (1) displayed weak inhibition of $\mathrm{LTC}_{4}$ release $\left(\mathrm{IC}_{50} 22.4 \mu \mathrm{M}\right)$. Leukotrienes including $\mathrm{LTC}_{4}$ are lipid mediators found elevated in various inflammatory diseases such as allergic rhinitis, asthma, and atopic dermitis. Currently, antileukotrienes are being prescribed for the treatment of asthma and have been approved for the treatment of allergic rhinitis. ${ }^{32}$ In the present study, compound $\mathbf{4}$ was found to possess the inhibitory activity and seemed to be a potential therapeutic effect on the aforementioned inflammatory diseases.

\section{Experimental Section}

General Procedures. Melting points were determined on a Kofler micro-ho tstage (uncorrected). Optical rotations were measured on a JASCO P-1020 polarimeter. UV spectra were measured on a Shimadzu UV-1601 UV-visible. The NMR spectra were recorded on a Varian Unity 400 FT-NMR spectrometers with the tetramethylsilane as an internal standard. Chemical shifts are presented in ppm. HRESIMS were measured on a Waters Q-Tof Premier mass spectrometer. Column chromatography (CC) was performed on silica gel (70 - 230 and 230 400 mesh, Merck), reverse-phase C18 gel (40 $\mu \mathrm{m}$, Nacalai, USA, Inc.). Thin layer chromatography (TLC) was performed on Kieselgel $60 \mathrm{~F}_{254}$ (Merck) or RP-18 $\mathrm{F}_{254 \mathrm{~s}}$ (Merck) plates. Spots were visualized by spraying $10 \%$ aqueous $\mathrm{H}_{2} \mathrm{SO}_{4}$ solution on the plates and heating them for $5 \mathrm{~min}$.

Plant Material. The fruits of Solanum nigrum were collected at a farm of Ggamajoong Korea Co. (Jeungpyeong, Korea) in July 2007. The plant material was identified by Dr. Joongku Lee (KRIBB), and a voucher specimen (01283) has been deposited at the Plant Extract Bank, Korea Research Institute of Bioscience and Biotechnology, Korea.

Extraction and Isolation. The fresh fruits of S. nigrum (15.0 $\mathrm{kg}$ ) was extracted with aqueous $\mathrm{EtOH}(50 \%)$ at room temperature $(20 \mathrm{~L} \times 3)$ to obtain $2 \mathrm{~kg}$ of the solid extract. The EtOH extract was suspended in $\mathrm{H}_{2} \mathrm{O}$ and extracted with EtOAc $(3 \mathrm{~L} \times$ $3)$ to give the EtOAc-soluble fractions $(97.0 \mathrm{~g})$. The EtOAcsoluble fraction $(97.0 \mathrm{~g})$ was chromatographed on a silica gel column eluted with a stepwise gradient of hexane and EtOAc to yield six fractions (Fr. A-F: $0.9 \mathrm{~g}, 2.4 \mathrm{~g}, 12.0 \mathrm{~g}, 18.7 \mathrm{~g}, 8.5 \mathrm{~g}$, $41.0 \mathrm{~g}$ each). Fr. B (2.4 $\mathrm{g}$ ) was subjected to a silica gel column chromatography eluted with a $\mathrm{CHCl}_{3}-\mathrm{MeOH}(100: 1)$ to give compounds $\mathbf{5}(28.0 \mathrm{mg}), \mathbf{7}(3.0 \mathrm{mg}), \mathbf{1 1}(5.0 \mathrm{mg})$ and $\mathbf{1 4}(3.0$ $\mathrm{mg})$. Compounds $4(3.3 \mathrm{~g})$ and $\mathbf{1 5}(84.0 \mathrm{mg})$ were purified from Fr. C (12.0 g) by a reversed-phase C-18 column chromatography using an isocratic solvent system $\mathrm{MeOH}-\mathrm{H}_{2} \mathrm{O}(1: 2)$. Fr. $\mathrm{D}(18.7 \mathrm{~g})$ was applied to a reversed-phase $\mathrm{C}-18$ column chromatography $\mathrm{MeOH}-\mathrm{H}_{2} \mathrm{O}(3: 1)$ and gave compound $9(1.7 \mathrm{~g})$. Fr. E (8.5 g) was chromatographed on a silica gel column, using $\mathrm{CHCl}_{3}-\mathrm{MeOH}-\mathrm{H}_{2} \mathrm{O}(70: 30: 4)$ as eluting solvent, to yield seven sub-fractions (Fr. E1-E7: $0.5 \mathrm{~g}, 0.9 \mathrm{~g}, 1.1 \mathrm{~g}, 2.7 \mathrm{~g}, 0.4 \mathrm{~g}$, $0.25 \mathrm{~g}, 2.0 \mathrm{~g}$ each). Fr. E3 $(1.1 \mathrm{~g})$ was further chromatographed on a reversed-phase $\mathrm{C} 18$ column $\mathrm{MeOH}-\mathrm{H}_{2} \mathrm{O}(1: 2)$ to give compounds $\mathbf{1}(8.5 \mathrm{mg}), \mathbf{1 2}(5.0 \mathrm{mg})$ and $\mathbf{1 3}(3.0 \mathrm{mg})$. Fr. E4 $(2.7 \mathrm{~g})$ was chromatographed on a reversed-phase $\mathrm{C} 18$ column using $\mathrm{MeOH}-\mathrm{H}_{2} \mathrm{O}(1: 3)$ to give compounds $3(15.0 \mathrm{mg}), \mathbf{6}$ $(5.8 \mathrm{mg})$, and $\mathbf{1 0}(6.0 \mathrm{mg})$. Compound $\mathbf{2}(17.0 \mathrm{mg})$ was isolated 
from Fr. E6 ( $0.25 \mathrm{~g})$ by a RP C-18 column eluted with $\mathrm{MeOH}-$ $\mathrm{H}_{2} \mathrm{O}(1: 2)$. Compound $8(5.9 \mathrm{~g})$ was obtained from Fr. F (41.0 g) by a silica gel column chromatography eluted with a solvent mixture of $\mathrm{CHCl}_{3}-\mathrm{MeOH}-\mathrm{H}_{2} \mathrm{O}(70: 30: 4)$.

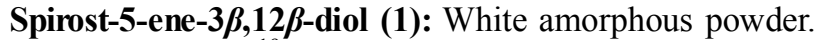
$\operatorname{mp} 283-284{ }^{\circ} \mathrm{C} .[\alpha]_{\mathrm{D}}^{19}-66.0^{\circ}(c=0.1, \mathrm{MeOH})$. UV $(\mathrm{MeOH})$ $\lambda_{\max }(\log \varepsilon): 203(3.80) \mathrm{nm}$. HR-negative ESI-MS $m / z: 415.2859$ $[\mathrm{M}-\mathrm{H}]^{-}$(calcd. for $\mathrm{C}_{26} \mathrm{H}_{39} \mathrm{O}_{4}, 415.2848$ ). ${ }^{1} \mathrm{H}$ - and ${ }^{13} \mathrm{C}-\mathrm{NMR}$ data see Table 1.

LTC $_{4}$ Release Assay. This assay was performed according to the literature. ${ }^{33}$ Rat basophilic leukemia (RBL-1) cells were plated in 96-well plates at a density of $1 \times 10^{5}$ cells/well. The test compounds were added and then incubated for 10 mins. Later, calcium ionophore A23187 was added and incubated further for 15 minutes. After incubation period, plates were centrifuged at $3000 \mathrm{rpm}$ and $4{ }^{\circ} \mathrm{C}$ for $10 \mathrm{~min}$. $\mathrm{LTC}_{4}$ in the supernatant of each well was measured using an ELISA kit (Cayman Chemical Co., USA) according to the manufacturer's recommended procedures. In brief, $50 \mu \mathrm{L}$ of supernatant, $\mathrm{LTC}_{4}$ acetylcholinetransferase, and $\mathrm{LTC}_{4}$ antiserum were added to each EIA well. Following incubation for $18 \mathrm{~h}$ at room temperature, the well was emptied and washed five times with washing buffer. Ellman's reagent $(200 \mu \mathrm{L})$ was added to each well, and the plate was placed on an orbital shaker for $1 \mathrm{~h}$ in the dark. The absorbance was measured at a wavelength of $405 \mathrm{~nm}$. Zileuton (Sequoia Research. Products Ltd, Oxen, UK) was used for a positive control.

Acknowledgments. This research was supported by a grant (PDM0500930) from the Plant Diversity Research Center of the 21st Century Frontier Research Program funded by the Ministry of Science and Technology of the Korean government and a grant of KRIBB Research Initiative Program (KGS2210 911).

\section{References and Notes}

1. Sultana, S.; Perwaiz, S.; Iqbal, M.; Athar, M. J. Ethnopharmacol. 1995, 45, 189.

2. Prashanth, K.; Shashidhara, S.; Kumar, M.; Sridhara, B. Fitoterapia. 2001, 72, 481.

3. An, H. J.; Kwon, K. B.; Cho, H. I.; Seo, E. A.; Ryu, D. G.; Hwang, W. J.; Yoo, S. J.; Kim, Y. K.; Hong, S. H.; Kim, H. M. Eur. J. Cancer Prev. 2005, 14, 345

4. Lee, S. J.; Lim, K. T. Toxicol. In Vitro. 2006, 20, 1088.

5. Zakaria, Z. A.; Sulaiman, M. R.; Morsid, N. A.; Aris, A.; Zainal,
H.; Pojan, N. H.; Kumar, G. H. Methods Find Exp Clin Pharmacol. 2009, 31, 81.

6. Heo, K. S.; Lim, K. T. J. Med. Food 2004, 7, 349.

7. Li, J.; Li, Q. W.; Gao, D. W.; Han, Z. S.; Li, K. Pharmazie 2008, $63,534$.

8. Li, J.; Li, Q. W.; Gao, D. W.; Han, Z. S.; Lu, W. Z. Phytother. Res. 2009, DOI: $10.1002 /$ ptr.2769

9. Jainu, M.; Devi, C. S. J. Ethnopharmacol. 2006, 104, 156.

10. Jeong, J. B.; Jeong, H. J.; Park, J. H.; Lee, S. H.; Lee, J. R.; Lee, H. K.; Chung, G. Y.; Choi, J. D.; Lumen, B. O. J. Agric. Food Chem. 2007, 55, 10707.

11. Lin, H. M.; Tseng, H. C.; Wang, C. J.; Lin, J. J.; Lo, C. W.; Chou, F. P. Chem. Biol. Interact. 2008, 171, 283.

12. Sarker, S. D.; Bartholomew, B.; Nash, R. J. Fitoterapia 2000, 71, 328.

13. Xing, X. C.; Ho, P.; Bourquin, G.; Yeh, L. A.; Cuny, G. D. Tetrahedron 2003, 59, 9961.

14. Baderschneider, B.; Winterhalter, P. J. Agric. Food Chem. 2001, 49, 2788.

15. Hu, K.; Jeong, J. H. Arch. Pharm. Res. 2006, 29, 563.

16. Ichikawa, M.; Ryu, K.; Yoshida, J.; Ide, N.; Kodera, Y.; Sasaoka, T.; Rosen, R. T. J. Agric. Food Chem. 2003, 51, 7313.

17. Ma, Z. Z.; Hano, Y.; Nomura, T.; Chen, Y. J. J. Nat. Prod. 2000, 63,390 .

18. Tamura, Y.; Hattori, M.; Konno, K.; Kono, Y.; Honda, H.; Ono, H.; Yoshida, M. Chemoecology 2004, 14, 113.

19. Yokozawa, T.; Zhou, J. J.; Sun, X. L.; Hattori, M.; Namba, T.; Tanaka, T.; Nishioka, I.; Oura, H. Natural Medicines 1994, 48, 330.

20. Dall'Acqua, S.; Innocenti, G.; Viola, G.; Piovan, A.; Caniato, R.; Cappelletti, E. M. Chem. Pharm. Bull. 2002, 50, 1499.

21. Chang, I. M.; Yun, H. S.; Yamasaki, K. Kor. J. Pharmacog. 1981, 12,12 .

22. Calis, I.; Kuruuzum, Uz. A.; Lorenzetto, P. A.; Ruedi, P. Phytochemistry 2002, 59, 451.

23. Cheng, Y. X.; Zhou, J.; Deng, S. M.; Tan, N. H. Planta Med. 2002, $68,91$.

24. Southwell, I. A.; Russell, M. F.; Maddox, C. D.; Wheeler, G. S. J. Chem. Ecol. 2003, 29, 83.

25. Fenn, P.; Durbin, R. D.; Kuntz, J. E. Phytochemistry 1977, 16, 899.

26. Heidelberger, M.; Jacobs, W. A. J. Am. Chem. Soc. 1919, 41, 1450.

27. Zhou, X.; He, X.; Wang, G.; Gao, H.; Zhou, G.; Ye, W.; Yao, X. J. Nat. Prod. 2006, 69, 1158.

28. Ori, K.; Mimaki, Y.; Mito, K.; Sashida, Y.; Nikaido, T.; Ohmoto, T.; Masuko, A. Phytochemistry 1992, 31, 2767.

29. Kingston, J. F.; Fallis, A. G. Can. J. Chem. 1982, 60, 820.

30. Kurata, K.; Taniguchi, K.; Shiraishi, K.; Suzuki, M. Phytochemistry 1990, 29, 3678.

31. Wakabayashi, T.; Kumonaka, Y.; Ichikawa, H.; Murota, S. J. P $1985 / 60152454$.

32. Duroudier, N. P.; Tulah, A. S.; Sayers, I. Allergy 2009, 64, 823.

33. Bickel, D.; Roder, T.; Bestmann, H. J.; Brune, K. Planta Med. 1994, 60, 318 . 